ÉGYPTE

monde arabe

\section{Égypte/Monde arabe}

12-13 | 1993

Une économie en transition

\title{
Une solution de rechange : notre projet islamique de développement
}

Al-Cha'b, $1^{\text {er }}$ août 1992

Muhammad 'Abd Allah Al-Chami

Traducteur : Samia Rizq

\section{OpenEdition}

\section{Journals}

Édition électronique

URL : https://journals.openedition.org/ema/1297

DOI : 10.4000/ema. 1297

ISSN : 2090-7273

\section{Éditeur}

CEDEJ - Centre d'études et de documentation économiques juridiques et sociales

\section{Édition imprimée}

Date de publication : 31 mars 1993

Pagination : 281-289

ISSN : 1110-5097

\section{Référence électronique}

Muhammad 'Abd Allah Al-Chami, « Une solution de rechange : notre projet islamique de

développement », Égypte/Monde arabe [En ligne], 12-13 | 1993, mis en ligne le 08 juillet 2008, consulté le 07 juillet 2022. URL : http://journals.openedition.org/ema/1297 ; DOl : https://doi.org/10.4000/ema. 1297

Ce document a été généré automatiquement le 7 juillet 2022.

Tous droits réservés 


\title{
Une solution de rechange : notre projet islamique de développement
}

\author{
Al-Cha'b, $1^{\text {er }}$ août 1992 \\ Muhammad 'Abd Allah Al-Chami \\ Traduction : Samia Rizq
}

1 Le gouvernement a enfin réussi à rassembler à l'Assemblée du Peuple quarante ou cinquante de ses députés pour leur soumettre le budget 1992-93, ainsi que le plan quinquennal 92-97 au moyen duquel le pouvoir prétend assurer le développement et la modernisation de l'Égypte jusqu'en 1997 et mettre le pays en mesure d'aborder le $\mathrm{XX}^{\mathrm{e}}$ siècle. Les députés présents ne représentaient que le dixième de la totalité des élus (468). Sachant que cette séance n'a pu recueillir que le dixième des voix des membres présents, cela signifie que ce plan n'a été approuvé que par... $1 \%$ de la population. Il s'agit donc du plan du gouvernement et des quarante députés du parti au pouvoir.

2 Leçon intéressante du point de vue politique. Comme le dit un proverbe anglais : "Vous pouvez tirer le cheval jusqu'à la rivière mais ne pouvez le forcer à en boire l'eau ». L'article 21 du projet de constitution de 1876 exige la présence du tiers des députés pour que l'Assemblée puisse valablement délibérer et prendre une décision (ce qui, dans notre cas, veut dire qu'à moins de 306 députés, le quorum n'est pas atteint). Voyez dans quelle fuite en avant nous nous trouvons...

Si nous examinons ce plan et les circonstances dans lesquelles il a été élaboré, publié et discuté, nous nous apercevons qu'il s'agit d'une des dizaines de « coquilles vides » que le gouvernement présente au peuple pour lui faire croire qu'une réforme est en cours et que l'avenir est plein d'espoir. En réalité, et selon le point de vue scientifique qui est le nôtre ici, c'est un plan de destruction de toute réflexion planificatrice en Égypte, un plan de limitation du développement de ce pays, une application insensée de la politique du FMI et des institutions qui en dépendent, avant-garde des puissances étrangères qui se préparent à dominer définitivement notre pays et à détruire les fruits d'un demi-siècle de lutte nationale, de libération et de renouveau, à faire passer le projet sioniste dans la région et à démolir le projet islamique. Avant d'analyser de près 
ce plan et ses orientations, il convient de passer en revue certains principes afin de mieux comprendre le rôle de la planification dans l'Égypte moderne.

4 En effet, l'opération de Suez, en 1956, était une tentative visant à porter un coup d'arrêt au développement de la défense égyptienne, une réponse à l'audacieuse action nationale que représentait la nationalisation du Canal, enfin un moyen d'enterrer le processus de planification. A peine l'Égypte avait-elle réagi en lançant son "plan géant » de 1960-65, que survenait, en 1967, la contre-attaque occidentalo-sioniste. Le processus de planification n'a donc pas eu le temps de reprendre son souffle, de se réorganiser pour lancer un deuxième plan reposant sur les résultats du premier. Cette attaque est survenue au moment où l'Égypte, encore perturbée, tentait de réorganiser son action nationale, de consolider la souveraineté du peuple et d'assimiler l'évolution sociale réalisée par le plan de 1960-65 : la nécessité se faisait sentir de renforcer cette évolution par un changement politique assurant au citoyen une plus grande liberté dans une patrie devenue libre, et préparant la prise en charge du pouvoir par la génération qui avait élaboré et exécuté le plan et qui s'était battue pour le défendre.

\section{Les jalons d'un plan national de défense et de développement}

5 La reconstruction du pays après 1967 et la préparation de la contre-attaque, couronnée par les batailles du Ramadan glorieux, sont le fruit d'un plan national pour la défense du développement. La force de l'attaque, le retrait de l'ennemi et la restitution du sol étaient le signe que se poursuivait la marche vers le développement. Un million de combattants concentrés sur les bords du canal et, derrière eux, neuf millions de travailleurs mobilisés sur les lieux de production constituaient l'avant-garde d'un peuple actif prêt à se battre pour le progrès. Dès la première semaine de la guerre de 1973, les puissances mondiales étaient préoccupées par un élément plus important que les résultats des combats-lesquels prédisaient déjà la destruction de l'arrogance sioniste et le sursaut du géant patriotique : les études internationales (britanniques, américaines, sionistes et autres) cherchaient à reconnaître les voies qu'emprunterait la victoire : comment l'Égypte, après avoir mobilisé toute cette force nationale dans une bataille d'une telle envergure, allait-elle l'employer une fois les armes déposées, une fois que des milliers de soldats seraient rentrés chez eux après avoir pris conscience de leur valeur et de l'histoire glorieuse de leur pays? Telle est la question qu'on se posait en Occident.

\section{La contre-attaque}

6 Ceci explique la contre-attaque que nous vivons aujourd'hui, conséquence concrète de la cinquième guerre israélo-arabe, à commencer par la politique d'ouverture (infitah), l'invasion de produits et capitaux étrangers, les dettes qui ont accablé le pays, la tutelle de la Banque mondiale et du FMI et le pillage des richesses intellectuelles, morales et matérielles de nos enfants; une politique qui tente d'instaurer une nouvelle classe de "Mamelouks ", qui dirige l'Égypte pour servir les intérêts américano-sionistes visant à dominer la région. Certes, 1967 était une contre-attaque de l'ennemi visant le plan de 
développement, et la guerre d'Octobre une contre-attaque égyptienne pour défendre le progrès et supprimer tout obstacle sur la voie du développement.

7 Cependant, la conjoncture internationale ayant changé et les progrès technologiques ayant ouvert au monde de nouveaux horizons, la guerre a pris elle aussi d'autres formes. La nouvelle arme de l'ennemi consiste à freiner le développement de l'Égypte en contrôlant le taux de croissance et en exploitant tout projet égyptien au profit de l'étranger, cela en le soumettant au système économique mondial dirigé par les ÉtatsUnis. Sans une bonne compréhension de ce qu'est véritablement cette arme, il est difficile de saisir le problème du développement et de la planification en Égypte, ainsi que des violentes attaques lancées contre l'action nationale.

\section{Le rôle du général al-Chazli}

8 Ce qui est vraiment regrettable, c'est que l'homme de génie qui a élaboré un contreplan égyptien pour défendre le développement et faire passer l'Égypte de la défaite à la victoire, cet homme est maintenant, tel un lion blessé, enfermé derrière les barreaux. Indépendamment de toutes considérations personnelles ou publiques relatives au mode de prise de décisions en Égypte, Sa'd al-Chazli a été à l'origine de toutes les actions entreprises pour préparer l'Égypte au combat, terrestre, aérien ou naval. Chef d'étatmajor des forces armées égyptiennes, il incarnait ce que les Égyptiens étaient capables d'offrir à leur patrie.

9 Tout Égyptien portant en lui l'amour de la patrie doit comprendre que ce sont tous ces grands hommes qui se trouvaient derrière les missiles, derrière les canons, dans les airs, sur les flots ou dans l'infanterie qui s'élançait vers la victoire ou vers la mort. Chacun de ces hommes était un Sa'd al-Chazli, combattant téméraire défendant corps et âme le développement de l'Égypte et sa modernisation.

10 Les jeunes posent aujourd'hui des questions fondamentales quant à l'utilité et à l'efficacité de la planification et quant aux caractéristiques du projet islamique de développement. Ils veulent savoir s'il peut devenir notre projet national, quelle est la valeur réelle du développement financé par des crédits étrangers, quel est le rôle de la Banque mondiale et des organisations qui s'y rattachent dans les plans de développement égyptiens. Ils s'interrogent également sur cet objectif de «doublement» du revenu national et sur l'état réel de l'économie égyptienne aujourd'hui, ainsi que sur la réalité des ressources et leur volume : suffisent-elles à la gestion et au financement du projet national de développement ? J'ai entre les mains le discours du gouvernement sur le plan 1992-97. La réponse à toutes ces questions vient en temps utile et permet une compréhension plus complète et une vision plus claire de la réalité.

\section{Caractéristiques du projet islamique}

11 Le projet islamique vise fondamentalement le développement continu de l'homme, qui doit se libérer et se préparer à peupler la terre. Dans la société islamique, le développement relève de la responsabilité commune. Dans la première société fondée par le Prophète - la paix de Dieu soit sur lui - à Médine, immédiatement après l'Hégire, le guide a voulu bâtir la nation sur le Coran et les hadith par le biais de l'éducation et de 
l'enseignement ; quant à l'action, elle repose sur la prédication, sa propagation et sa défense.

12 Sous le règne des quatre califes "bien guidés ", cet État naissant réalisait un taux annuel de croissance de $25 \%$ et, selon certaines estimations, les revenus de cette société doublaient tous les deux ans. Au départ, il n'y avait qu'une mosquée construite par le Prophète, où se rassemblaient les hommes de valeur. De cette mosquée est sortie une armée - « Je ne trouve aucune monture à vous donner » - que l'État a lancée vers le nord, l'est et l'ouest à la conquête des deux plus grands empires. En moins d'un quart de siècle, le plus grand État jamais édifié par l'homme naquit avec la grâce de Dieu «La main de Dieu est posée sur leurs mains ».

13 Cette communauté purifiée par l'aumône a pu, grâce à des offrandes jamais égalées dans l'histoire, soigner les maux de la société. Chacun s'évertuait à « dépenser pour la cause de Dieu » afin de contribuer au développement de l'État naissant : c'était là un honneur auquel tout le monde aspirait.

14 La charité, jointe à la foi, rendait le musulman digne de faire partie de cette communauté, dont le développement reposait sur l'enseignement et l'éducation de la foi, et la bienfaisance concrétisée par l'aumône légale (zakât) et l'acquittement de droits sur les recettes (haqq al-mâl). Ainsi, tout individu jouissait d'une protection sociale dans cet État fondé sur le principe de la consultation et sur une loi fondamentale consistant à « ordonner le bien et interdire le mal ». Un État où il était « interdit à un musulman de porter atteinte à un autre musulman dans son sang, son honneur ou ses biens » et où "si un homme meurt de faim, tous les siens en seront tenus pour responsables"; où «si un mulet tombe sur la route, le gouverneur aura des comptes à rendre pour avoir négligé l'entretien de la route ". C'était une société mobilisée en permanence par le travail et l'union pour la cause de Dieu... Tel est le sens du développement. Que la jeunesse le sache.

\section{Le projet étranger}

En Égypte, comme dans tout pays musulman qui a eu le malheur d'être envahi, cohabitent deux projets contradictoires : un projet étranger et un projet national.

16 Le projet étranger, c'est l'occupation armée, l'invasion culturelle par le biais de l'abolition de l'identité et de la transformation de l'entité nationale, du déracinement, de l'ébranlement des croyances religieuses; c'est une domination économique visant à transformer le pays en marché pour les produits étrangers, en port pour les flottes étrangères, en plaque tournante de l'empire économique mondial.

17 C'est également un projet de développement contrôlé prévoyant un taux de croissance à ne pas dépasser; c'est le projet des «Mamelouks" de tous les temps et de leurs partisans, qui dominent le pays par l'intermédiaire d'une classe dirigeante "fabriquée " par l'occupation; c'est le projet de la franc-maçonnerie internationale, des Rotary et Lyons Clubs, de la sédition confessionnelle, des pots-de-vin et de la corruption générale, de l'affaiblissement de l'armée et du contrôle de l'armement; un projet qui tient le peuple à l'écart de la technologie et de l'information; un projet où l'enseignement est "réservé aux cols blancs", où les forces de l'ordre sont réquisitionnées pour défendre les intérêts étrangers, châtier les nationalistes, soumettre le pays à la politique de la carotte et du bâton. Crédits et subventions sont 
accordés en échange de concessions ou en cas de besoin urgent exposant le pays au danger. Ainsi, entre les efforts qu'ils déploient pour leur développement et ceux qu'il leur faut déployer pour s'acquitter de leurs dettes extérieures, ces pays s'étiolent sans pouvoir ni se développer véritablement ni se libérer du joug étranger et jouir de l'indépendance.

\section{Le projet égyptien}

18 À l'opposé, le projet national: le projet de 'Umar Makram, de Ahmad 'Urabi et de la révolution de 1919; le projet du mouvement mené par Tal'at Harb et de l'éveil islamique issu d'Égypte, du mouvement du 23 juillet, de la «traversée » du Ramadan glorieux. L'objectif du projet national, c'est de construire l'Égypte de l'industrie et de l'agriculture modernes, l'Égypte de la civilisation qui occupe une place de choix dans toute la région et qui protège les deux villes saintes...

19 C'est un projet de développement global reposant sur l'utilisation des ressources nationales et le refus de la domination des crédits étrangers; c'est le projet de tous les Égyptiens - musulmans et coptes; c'est le projet du génie, de la science, de la technologie et de l'administration égyptienne; c'est le projet de l'union de l'Égypte avec le Soudan, de l'application de la chari'a en matière de gouvernement et de finances ; c'est le panarabisme islamique. Le projet national, c'est encore le principe de la consultation et du respect des droits de l'homme conformément à l'islam ; projet de gouvernement exclusivement local dans lequel le peuple est gouverné par le peuple; c'est l'aumône légale, les droits sur les recettes et les dons, non la perception d'impôts ; un projet où le peuple, propriétaire du secteur public, est responsable du développement.

20 Le projet national exige enfin que tout citoyen participe au développement de l'Égypte et du Soudan et que soit maintenu un taux de croissance annuel d'au moins $15 \%$ afin de sortir du sous-développement. Par conséquent, le projet national rejette toute politique de développement financée par des crédits étrangers.

21 Au cours de son histoire, ce projet a dû subir deux rudes épreuves : la première en 1876, avec la déclaration de la faillite de l'Égypte suivie de l'occupation du pays et, en 1882, de la perte de son indépendance; la deuxième en 1976, lorsqu'au niveau mondial l'Égypte fut de nouveau déclarée en faillite et qu'elle dut adhérer au Club de Paris. Elle fut alors soumise à la tutelle de la Banque mondiale et du FMI.

Lorsqu'en 1851, Rachid Pacha avait demandé un crédit de 55 millions de francs auprès d'une banque franco-britannique, le sultan s'y était opposé : «Si, cette année, nous empruntons une piastre, l'année prochaine nous en emprunterons davantage: l'endettement est une voie sans fin». Cependant, sous la pression des besoins de modernisation et des dépenses militaires qui lui étaient « imposés » dans le cadre d'un mouvement international dirigé par les usuriers juifs et les états qui convoitaient l'empire des califes, le sultan finit par demander un crédit de $3.815 .000 £$ au taux d'intérêt de $6 \%$.

L'établissement britannique qui conduisit l'opération perçut $1.300 .087 £$ à titre d'honoraires, et la somme reçue par le Trésor public se trouva donc réduite à $2.514 .913 £$ (soit $66 \%$ du montant total du prêt). Le budget de l'Égypte fut hypothéqué pour garantir le remboursement, alors que les coffres des hauts responsables 
regorgeaient d'or. Mais, telle est la volonté de Dieu lorsqu'il entend anéantir une nation : moins de vingt ans plus tard, l'État ottoman était en faillite !

Puis, en 1876, lorsqu'à la suite de la déclaration de faillite de l'Égypte, deux ministres étrangers furent nommés au cabinet Noubar, les dettes furent réévaluées : alors que le total des sommes versées au Trésor égyptien s'élevait à environ 50 millions de livres, la somme totale due était de l'ordre de 100 millions de livres à cause des honoraires, potsde-vin et frais divers qui étaient venus s'y ajouter. A la fermeture de la Caisse des crédits en 1943, le montant total des sommes versées par l'Égypte avait atteint 250 millions de livres ! Et après tout cela, on continue à emprunter !

\section{L'emprunt, c'est la faillite}

Voilà pourquoi une des constantes du projet national est de considérer qu'emprunt et faillite sont les deux faces d'une même pièce. Avant la guerre du Golfe, les dettes extérieures de l'Égypte s'élevaient à plus de 200 milliards LE. Le service de la dette consomme plus de $20 \%$ du budget de l'État et plus de $30 \%$ du total des recettes de l'exportation. La dette publique atteint donc plus de 3000 livres par an et par tête. Dans le projet national, le "développement importé » n'a pas de sens... C'est une pure invention... Les crédits extérieurs ne sont que le « cheval de Troie» qui permet aux étrangers de pénétrer dans le pays pour freiner le développement, imposer leurs conditions et faire tomber l'État dans leur piège.

Quant à l'endettement véritable de l'Égypte, personne ne le connaît en dehors de Washington et de Tel Aviv. Les chiffres avancés sont tellement contradictoires que le chef de l'État, le chef du gouvernement et le gouverneur de la Banque centrale sont tournés en dérision par la presse internationale. Certaines dettes sont telles que le gouvernement craint d'en dévoiler l'ampleur (certains Égyptiens se souviennent peutêtre que l'Égypte a continué à verser un impôt à l'État ottoman jusqu'aux années soixante, alors que la chute de l'Empire remonte à 1923...). Les rapports de la Banque mondiale et des institutions qui en dépendent montrent que les crédits accordés à l'Égypte ne sont rentabilisés qu'à $40 \%$ maximum, voire même $20 \%$ dans certains cas.

Il est mondialement connu qu'en dépit de l'endettement continuel de l'Égypte, le flux monétaire égyptien s'écoule toujours en direction de l'étranger, sans compter la fuite des capitaux privés qui n'atteint pas moins de 5 milliards de livres par an. Autrement dit, les comptes égyptiens à l'étranger sont de l'ordre de 50 à 100 milliards de dollars (soit 2 à 4 fois la dette extérieure). Le projet sioniste qui se dresse contre le projet national consiste à utiliser les fonds arabes sous contrôle financier international pour régler les dettes de l'Égypte, piller le secteur public et soumettre définitivement le pays à la domination étrangère. En participant à la guerre du Golfe, l'Égypte avait obtenu la promesse de l'annulation de toutes ses dettes. Or, après ces opérations, a été prélevée sur les fonds arabes une somme suffisante pour régler la moitié de la dette égyptienne ; quant à l'autre moitié, elle est maintenant soumise à la politique de la carotte et du bâton.

Voilà comment l'Égypte est gouvernée, tant sur le plan politique que sur le plan économique : la Banque mondiale et le FMI planifient et font pression, et les biens arabes ne servent qu'au chantage politique. Et dans cette effroyable spirale, l'Égypte perd son indépendance politique et son poids dans la région et sur la scène arabe et islamique. Lorsque l'histoire révélera la manière dont l'Égypte est tombée dans le piège 
de l'endettement, les Égyptiens seront humiliés au plus profond d'eux-mêmes et réaliseront vraiment la portée de la contre-attaque américano-sioniste et européenne lancée comme une vengeance après la glorieuse victoire de Ramadan 73.

Un groupe de responsables égyptiens s'est récemment rendu à Bruxelles pour mendier une aide... Certains de leurs hôtes avaient connu l'Égypte avant 1967 et ne parvenaient pas à croire que les hommes qui étaient en face d'eux étaient les mêmes que ceux qui avaient soulevé le monde entier contre l'Europe et l'Amérique, dirigé le mouvement de libération nationale et aspiraient alors au développement global de leur pays... Au cours de la réunion récente d'un comité du Sénat américain, on a demandé à l'ambassadeur d'Égypte : «Comment justifiez-vous l'aide américaine à l'Égypte? » Et l'ambassadeur de répondre: "Elle a pour but de nous aider à établir la stabilité en Égypte, car c'est de notre stabilité que dépend celle de tout le Moyen-Orient ». En 1950, le journal égyptien Al-Akhbâr rapportait qu'un Pacha s'était rendu à Londres pour prier les Anglais de quitter l'Égypte afin que le communisme ne s'y répande pas...

Il faut nous libérer de la domination du FMI. Financer le gouvernement par des crédits étrangers est un crime, à l'égard du présent comme de l'avenir. L'économie égyptienne est capable de s'acquitter de ses obligations. Reste à savoir comment! Les Égyptiens doivent être informés de la situation économique réelle de leur pays, des possibilités latentes dont il dispose et de ce que cette économie a permis de réaliser au cours de quarante années de libération et de lutte nationale.

\section{INDEX}

Mots-clés : projet islamique de développement 